Proc. 13th International School on Theoretical Physics: Symmetry and Structural Properties of Condensed Matter

\title{
The Example of Using the Schur-Weyl Duality in One-Dimensional Hubbard Model
}

\author{
D. JAKUBCZYK ${ }^{a, *}$ AND P. JAKUBCZYK ${ }^{b}$ \\ ${ }^{a}$ Faculty of Mathematics and Applied Physics, Rzeszów University of Technology, \\ al. Powstańców Warszawy 12, 35-959 Rzeszów, Poland \\ ${ }^{b}$ Faculty of Mathematics and Natural Sciences, University of Rzeszów, T. Rejtana 16A, 35-959 Rzeszów, Poland
}

\begin{abstract}
The one-dimensional Hubbard model is discussed assuming periodic boundary conditions and the half-filling case. The Schur-Weyl duality is applied to the chain consisting of six nodes with dual actions of the unitary and symmetric groups taken separately in the spin and pseudo-spin space.
\end{abstract}

DOI: 10.12693/APhysPolA.135.1252

PACS/topics: 75.10.Pq, 03.65.Aa, 03.65.Fd

\section{Introduction}

The Hubbard model is one of the most fundamental one-dimensional model of interacting particles in a lattice introduced to tackle the behaviour of correlated electrons in solid. John Hubbard [1] (1931-1980) found the model to be the simplest that produces both a metallic and an insulating states of approximate behaviour of interacting electrons in a solid, depending on the value of on-site repulsion $u$. One of the most successful descriptions of electrons in solids is though band theory. It is based on reducing many-body interactions to an effective one-body description. The Hubbard model became especially important as it showed that for half-filling the Mott transition is reproduced, that could not be understood in terms of conventional band theory. The Hubbard model is an extension of the so-called tight-binding model, where electrons can hop between lattice sites as independent particles.

The aim of the calculations is to determine the eigenbasis adopted to the spin and the pseudo-spin symmetries for the case of the one-dimensional Hubbard model with $N$ atoms using the Schur-Weyl duality (SWD) [2]. SWD was introduced by Schur [3] and then further developed by Weyl [4], who showed that the Young symmetrizators of symmetric groups can be used to obtain irreducible representations of a unitary group. This approach leads for the half-filling case to significance reduction of the eigenproblem of the one-dimensional Hubbard Hamiltonian.

\section{The model}

The dynamics of the finite set of interacting electrons, occupying the one-dimensional chain, consisted of $N$ atoms, can be described by the Hubbard Hamiltonian in the following form

*corresponding author; e-mail: djak@prz.edu.pl

$$
H=t \sum_{i \in \tilde{2}} \sum_{j \in \tilde{N}}\left(c_{j i}^{\dagger} c_{j+1 i}+c_{j+1 i}^{\dagger} c_{j i}\right)+u \sum_{j \in \tilde{N}} n_{j+} n_{j-},(1)
$$

where $\tilde{N}=\{j=1,2, \ldots, N\}$ denotes the set of atoms of the chain, $\tilde{2}=\{i=+,-\}, n_{j i}=c_{j i}^{\dagger} c_{j i}$, and finally $c_{j i}^{\dagger}, c_{j i}$ are the canonical Fermi operators, that is creation and anihilation operators of electron of spin $i$, on the site $j$. The electron hopping in the Hubbard Hamiltonian can only take place between nearest-neighbour sites, and all hopping processes have the same kinetic energy.

The set of all linearly independent vectors called electron configurations $[5,6]$ provides the initial, orthonormal basis of the Hilbert space $\mathcal{H}$. These configurations are defined by the following mapping

$$
f: \tilde{N} \longrightarrow \tilde{4},
$$

and constitute the $N$-sequences of the elements from the set $\tilde{4}=\{ \pm, \emptyset,+,-\}$ as follows

$$
\begin{aligned}
& |f\rangle=|f(1) f(2) \ldots f(N)\rangle=\left|i_{1} i_{2} \ldots i_{N}\right\rangle, \\
& i_{j} \in \tilde{4}, \quad j \in \tilde{N},
\end{aligned}
$$

where $\emptyset$ denotes the empty node, + and - stand for onenode spin projection equal to $\frac{1}{2}$ and $-\frac{1}{2}$, respectively, \pm denotes the double occupation of the one node by two electrons with different spin projections, with

$$
\tilde{4}^{\tilde{N}}=\{f: \tilde{N} \longrightarrow \tilde{4}\}, \quad \mathcal{H}=\operatorname{lc}_{\mathbb{C}} \tilde{4}^{\tilde{N}} .
$$

\section{Symmetries of the model}

Since the periodic boundary condition are assumed [7], the Hamiltonian (1) has the obvious translational symmetry $\left(c_{N+1 i}=c_{1 i}\right)$. This means that one-particle Hamiltonian of the form (1) is completely diagonalised by a Fourier transformation in the form

$$
c_{k}^{\dagger}=\frac{1}{\sqrt{N}} \sum_{j \in \tilde{N}} \exp (\mathrm{i} 2 \pi k j / N) c_{j}^{\dagger},
$$

where

$$
k=0, \pm 1, \ldots, \begin{cases} \pm(N / 2-1), N / 2, & \text { for } N \text { even } \\ \pm(N-1) / 2, & \text { for } N \text { odd }\end{cases}
$$


labels the irreducible representations $[5,8]$ (irreps) $\Gamma_{k}(j)=\sum_{j \in \tilde{N}} \exp (\mathrm{i} 2 \pi k j / N), j \in \tilde{N}$ of the translational symmetry group $C_{N}$.

Apart from the cyclic symmetry, system reveals many other, among them, for the half-filling of the electrons, two independent $\mathrm{SU}(2)$ symmetries [7, 9], that is $\mathrm{SU}(2) \times \mathrm{SU}(2)$ in the spin and pseudo-spin space. This symmetry involves spin and charge degrees of freedom, and is related with four elementary excitation, that is spinon $\frac{1}{2}$, spinon $-\frac{1}{2}$, with respect to the spin, and holon, antiholon, with respect to the charge. The set $\tilde{4}=\{ \pm, \emptyset,+,-\}$ can be decomposed into two subsets, where first $\tilde{2^{\prime}}=\{ \pm, \emptyset\}$ is related with the left factor of the direct product $\mathrm{SU}(2) \times \mathrm{SU}(2)$ of the unitary groups, and the second set $\tilde{2}=\{+,-\}$ is related with the right factor, reflecting the invariance of $H$ under the spin rotation. Thus, one has two sets of generators, $\left\{S_{z}, S^{+}, S^{-}\right\}$ and $\left\{J_{z}, J^{+}, J^{-}\right\}$, for spin and charge, respectively.

\section{The Schur-Weyl duality for one-dimensional Hubbard model in the case of half-filling}

The action

$$
A: \Sigma_{N} \times \tilde{4}^{\tilde{N}} \longrightarrow \tilde{4}^{\tilde{N}}
$$

of the symmetric group $\Sigma_{N}[5]$ on the set $\tilde{4}^{\tilde{N}}$ provides the orbits $\mathcal{O}_{\mu}$ of the group $\Sigma_{N}$ labeled by the weight $\mu$, given as the sequence of non-negative integers $\mu=$ $\left(\mu_{1}, \mu_{2}, \mu_{3}, \mu_{4}\right)$, where the consecutive $\mu_{i}$ denote the number of $\pm, \emptyset,+$ and - in the electron configuration, respectively, where $\sum_{i \in \tilde{4}} \mu_{i}=N$ and $\mu_{i}=\left|\left\{i_{j}=i \mid j \in \tilde{N}\right\}\right|$, $i \in \tilde{4}$. Such an orbit is invariant under the action of the symmetric group $\Sigma_{N}$ and forms the carrier space of the transitive representation $R^{\Sigma_{N}: \Sigma^{\mu}}$, with the stabilizer $\Sigma^{\mu}$ being the Young subgroup $\Sigma^{\mu}=\Sigma_{\mu_{1}} \times \Sigma_{\mu_{2}} \times \Sigma_{\mu_{3}} \times \Sigma_{\mu_{4}}$, where $\times$ denotes the Cartesian product.

Since there are two independent $\mathrm{SU}(2)$ symmetries one can consider the action of the symmetric group $\Sigma_{N}$ - in context of the Schur-Weyl duality $[2,10]$ — separately in the spin and pseudo-spin space in order to obtain the total spin $S$ and the total pseudo-spin $J$. This observation holds for the system of any number $N$ of atoms and provides two symmetric group $\Sigma_{N^{\prime}}$ and $\Sigma_{N^{\prime \prime}}$ in the spin and pseudo-spin space, respectively. The actions

$$
A: \Sigma_{N} \times \tilde{4}^{\tilde{N}} \longrightarrow \tilde{4}^{\tilde{N}}, \quad B: \mathrm{U}(4) \times \tilde{4}^{\tilde{N}} \longrightarrow \tilde{4}^{\tilde{N}}
$$

are replaced by

$$
A^{\prime}: \Sigma_{N}^{\prime} \times \tilde{2}^{\tilde{N}^{\prime}} \longrightarrow \tilde{2}^{\tilde{N}^{\prime}}, \quad B^{\prime}: \mathrm{SU}(2) \times \tilde{2}^{\tilde{N}^{\prime}} \longrightarrow \tilde{2}^{\tilde{N}^{\prime}},
$$

in the spin space $\mathcal{H}_{s}=\operatorname{lc}_{\mathbb{C}} \tilde{2}^{\tilde{N}^{\prime}}=h_{s}^{\otimes N^{\prime}}$, where $h_{s} \cong \mathbb{C}_{2}$ denotes the one-node spin space, and

$$
A^{\prime \prime}: \Sigma_{N}^{\prime \prime} \times \tilde{2}^{\tilde{N}^{\prime \prime}} \longrightarrow \tilde{2}^{\tilde{N}^{\prime \prime}}, \quad B^{\prime \prime}: \mathrm{SU}(2) \times \tilde{2}^{\tilde{N}^{\prime \prime}} \longrightarrow \tilde{2}^{\tilde{N}^{\prime \prime}},
$$

in the pseudo-spin space $\mathcal{H}_{p}=l c_{\mathbb{C}} \tilde{2}^{\tilde{N}^{\prime \prime}}=h_{p}^{\otimes N^{\prime \prime}}$, where $h_{p} \cong \mathbb{C}_{2}$ denotes the one-node pseudo-spin space. The spin and pseudo-spin spaces are isomorphic with Hilbert space of the one-dimensional Heisenberg model for the case of $N^{\prime}$ and $N^{\prime \prime}$ nodes of the spin chain, respectively.
Let us define some initial Hilbert space as follows

$$
\begin{aligned}
& \mathcal{H}_{i n t}=\bigoplus_{\left(\tilde{N}^{\prime}, \tilde{N}^{\prime \prime}\right)}\left(\mathcal{H}_{s} \otimes \mathcal{H}_{p}\right), \\
& \tilde{N}^{\prime} \cup \tilde{N}^{\prime \prime}=\tilde{N}, \tilde{N}^{\prime} \cap \tilde{N}^{\prime \prime}=\emptyset,
\end{aligned}
$$

where $N^{\prime}$ and $N^{\prime \prime}$ denotes the cardinalities of the sets $\tilde{N}^{\prime}$ and $\tilde{N}^{\prime \prime}$, respectively, and $\left(\tilde{N}^{\prime}, \tilde{N}^{\prime \prime}\right)$ stands for the pair of these two sets-each taken in ascending order. The last equations means that from now on we will label the Hilbert space (4) by $\mathcal{H}_{\text {int }}$. The space (11) can be decomposed with respect to the number of electrons in the system

$$
\mathcal{H}_{\text {int }}=\bigoplus_{N_{e}=0}^{2 N} \mathcal{H}^{N_{e}}
$$

and further with respect to the number of electron with fixed spin projection

$$
\mathcal{H}^{N_{e}}=\bigoplus_{\left(N_{+}, N_{-}\right)=(0,0)} \mathcal{H}_{\left(N_{+}, N_{-}\right)}^{N_{e}}, \quad N_{+}+N_{-}=N_{e},
$$

where $N_{+}$and $N_{-}$denote the number of electrons with spin projection equal to $1 / 2$ and $-1 / 2$, respectively. Since the symmetry $\mathrm{SU}(2) \times \mathrm{SU}(2)$ holds only for halffilling case the proper Hilbert space $\mathcal{H}$ for the case considered in the present paper is the subspace $\mathcal{H}^{N_{e}=N} \equiv \mathcal{H}$ of the initial space (11).

The actions (9) and (10) provide two transitive representations $R^{\Sigma_{N^{\prime}}:\left(\Sigma_{\mu_{3}} \times \Sigma_{\mu_{4}}\right)}$ and $R^{\Sigma_{N^{\prime \prime}}:\left(\Sigma_{\mu_{1}} \times \Sigma_{\mu_{2}}\right)}$ in the spin and pseudo-spin space, respectively, where $\Sigma^{\mu^{\prime}}=$ $\Sigma_{\mu_{3}} \times \Sigma_{\mu_{4}}$ and $\Sigma^{\mu^{\prime \prime}}=\Sigma_{\mu_{1}} \times \Sigma_{\mu_{2}}$. Each transitive representation decomposes as

$$
R^{\Sigma_{N^{\prime}}: \Sigma^{\mu^{\prime}}} \cong \sum_{\lambda^{\prime} \geq \mu^{\prime}} K_{\lambda^{\prime} \mu^{\prime}} \Delta^{\lambda^{\prime}}=\sum_{\lambda^{\prime} \geq \mu^{\prime}} \Delta^{\lambda^{\prime}},
$$

into irreps of the symmetric group $\Sigma_{N^{\prime}}$, with the partition $\lambda^{\prime} \vdash N^{\prime}$ defining the shape of the corresponding irrep $\Delta^{\lambda^{\prime}}$, where $K_{\lambda^{\prime} \mu^{\prime}}$ are the famous Kostka numbers, equal to 1 in case of two-dimensional one-node space, the sum runs over all partitions $\lambda^{\prime}$ of $N^{\prime}$ which are not smaller than $\mu^{\prime}$ in the dominance order, and $N^{\prime}$ denotes the number of appropriate one-node spin spaces $h_{s}$. For the pseudo-spin space with $N^{\prime \prime}$ number of appropriate one-node pseudo-spin spaces $h_{p}$ by analogy to (14) the following decomposition holds

$$
R^{\Sigma_{N^{\prime \prime}}: \Sigma^{\mu^{\prime \prime}}} \cong \sum_{\lambda^{\prime \prime} \geq \mu^{\prime \prime}} K_{\lambda^{\prime \prime} \mu^{\prime \prime}} \Delta^{\lambda^{\prime \prime}}=\sum_{\lambda^{\prime \prime} \geq \mu^{\prime \prime}} \Delta^{\lambda^{\prime \prime}},
$$

into irreps of the symmetric group $\Sigma_{N^{\prime \prime}}$, with the partition $\lambda^{\prime \prime} \vdash N^{\prime \prime}$ defining the shape of the corresponding irrep $\Delta^{\lambda^{\prime \prime}}$.

\section{The example of the chain consisted of six nodes}

In the present paper we examine the example of the chain with six nodes $N=6$ at the half-filling, thus the weights are as follows 


\begin{tabular}{ccccc}
$N^{\prime}$ & $N^{\prime \prime}$ & $N_{+}$ & $N_{-}$ & $\mu$ \\
\hline 6 & 0 & 6 & 0 & $(0,0,6,0)$ \\
& & 5 & 1 & $(0,0,5,1)$ \\
& & 4 & 2 & $(0,0,4,2)$ \\
& & 3 & 3 & $(0,0,3,3)$ \\
& & 2 & 4 & $(0,0,2,4)$ \\
& & 1 & 5 & $(0,0,1,5)$ \\
& & 0 & 6 & $(0,0,0,6)$ \\
\hline 4 & 2 & 5 & 1 & $(1,1,4,0)$ \\
& & 4 & 2 & $(1,1,3,1)$ \\
& & 3 & 3 & $(1,1,2,2)$ \\
& & 2 & 4 & $(1,1,1,3)$ \\
& & 1 & 5 & $(1,1,0,4)$ \\
\hline 2 & 4 & 4 & 2 & $(2,2,2,0)$ \\
& & 3 & 3 & $(2,2,1,1)$ \\
& & 2 & 4 & $(2,2,0,2)$ \\
\hline 0 & 6 & 3 & 3 & $(3,3,0,0)$
\end{tabular}

The dimension of the initial Hilbert space $\mathcal{H}_{\text {int }}$ given by the Eq. (11) for the case of the chain consisted of six nodes $N=6$ is equal to $4096=4^{6}$. The dimension of the proper Hilbert space given as the subspace $\mathcal{H}^{N_{e}}=N=6$ of the initial Hilbert space can be calculated as follows

$$
\mathcal{H}=\mathcal{H}^{N_{e}=6}=\bigoplus_{\left(N_{+}, N_{-}\right)=(0,0)} \mathcal{H}_{\left(N_{+}, N_{-}\right)}^{N_{e}=6},
$$

$$
N_{+}+N_{-}=6 \text {. }
$$

Thus the dimension of the Hilbert space $\mathcal{H}$ is equal to

$$
\begin{aligned}
& \operatorname{dim} \mathcal{H}=\operatorname{dim} \mathcal{H}_{(6,0)}^{6}+\operatorname{dim} \mathcal{H}_{(5,1)}^{6}+\operatorname{dim} \mathcal{H}_{(4,2)}^{6} \\
& \quad+\operatorname{dim} H_{(3,3)}^{6}+\operatorname{dim} \mathcal{H}_{(2,4)}^{6}+\operatorname{dim} \mathcal{H}_{(1,5)}^{6}+\operatorname{dim} \mathcal{H}_{(0,6)}^{6} \\
& \operatorname{dim} \mathcal{H}=1+36+225+400+225+36+1=924
\end{aligned}
$$

The result above can be written as follows

$$
2^{6}+\left(\begin{array}{l}
2 \\
1
\end{array}\right)\left(2^{4}\right)\left(\begin{array}{l}
6 \\
4
\end{array}\right)+\left(\begin{array}{l}
4 \\
2
\end{array}\right)\left(2^{2}\right)\left(\begin{array}{l}
6 \\
2
\end{array}\right)+\left(\begin{array}{l}
6 \\
3
\end{array}\right),
$$

since the multiplicity of deploying of $N^{\prime}$ spin atoms and $N^{\prime \prime}$ pseudo-spin atoms on the chain consisted of $N=N^{\prime}+N^{\prime \prime}$ atoms is equal to

$$
\tau=\left(\begin{array}{c}
N \\
N^{\prime}
\end{array}\right)=\left(\begin{array}{c}
N \\
N^{\prime \prime}
\end{array}\right) \text {. }
$$

The decomposition of the transitive representations of the actions (9) and (10) of the symmetric group $\Sigma_{6}$ into irreducible representations provides the irreducible basis with specified values of total spin $S$ and the total pseudospin $J$. For example, the total number of states for the case of $S_{z}=1$ and $J_{z}=0$ (the ninth row of the summary (16)) can be calculated as follows

$$
\begin{aligned}
& \operatorname{dim}\left[\left(R^{\left\{1^{2}\right\}} \otimes R^{\{31\}}\right)\right] \times \tau= \\
& \quad \operatorname{dim}\left[\left(\Delta^{\{2\}} \oplus \Delta^{\left\{1^{2}\right\}}\right) \otimes\left(\Delta^{\{4\}} \oplus \Delta^{\{31\}}\right)\right] \times \tau=8 \times \tau
\end{aligned}
$$

where the first and the second transitive representations correspond to the pseudo-spin and the spin space, respectively, since $N^{\prime \prime}=2$ and $N^{\prime}=4$. The multiplicity of deploying of $N^{\prime}$ spin atoms and $N^{\prime \prime}$ pseudo-spin atoms on the chain consisted of $N=N^{\prime}+N^{\prime \prime}$ atoms is equal to $\tau=15$, thus the number of states for the considered case is equal to 120 and together with the number of states for $N^{\prime}=6\left(N^{\prime \prime}=0\right)$ and $N^{\prime}=2\left(N^{\prime \prime}=4\right)$ for the same $S_{z}$ and $J_{z}$ contributes to the number 225 of the Eq. (18).

\section{Conclusions}

We presented the application of the Schur-Weyl duality in the one-dimensional Hubbard model in the case of half-filling for the example of six atoms. We introduced the spin and pseudo-spin space in order to obtain the total spin $S$ and the total pseudo-spin $J$. We used the concept of initial Hilbert space which provides the proper Hilbert space of the considered system as its subspace. The calculations are significant since the obtained results lead to a significant reduction in the size of the Hubbard Hamiltonian.

\section{References}

[1] J. Hubbard, Proc. R. Soc. A 276, 238 (1963).

[2] D. Jakubczyk, P. Jakubczyk, J. Math. Phys. 59, 023504 (2018).

[3] I. Schur, in: Gesammelte Abhandlungen, Vol. III, Springer, Berlin 1973, p. 68, 1927 (in German).

[4] H. Weyl, The Classical Groups. Their Invariants and Representations, Princeton UP, Princeton 1946.

[5] D. Jakubczyk, P. Jakubczyk, Centr. Eur. J. Phys. 10, 906 (2012).

[6] D. Jakubczyk, P. Jakubczyk, Acta Phys. Pol. B 42, 1825 (2011)

[7] F.H.L. Essler, H. Frahm, F. Göhmann, A. Klümper, V.E. Korepin, The One-Dimensional Hubbard Model, Cambridge University Press, 2005.

[8] B. Lulek, D. Jakubczyk, Centr. Eur. J. Phys. 1, 132 (2003).

[9] M. Cuoco et al., Int. J. Mod. Phys. B 11, 2511 (1997).

[10] P. Jakubczyk, T. Lulek, D. Jakubczyk, B. Lulek, J. Phys. Conf. Series 30, 203 (2006). 